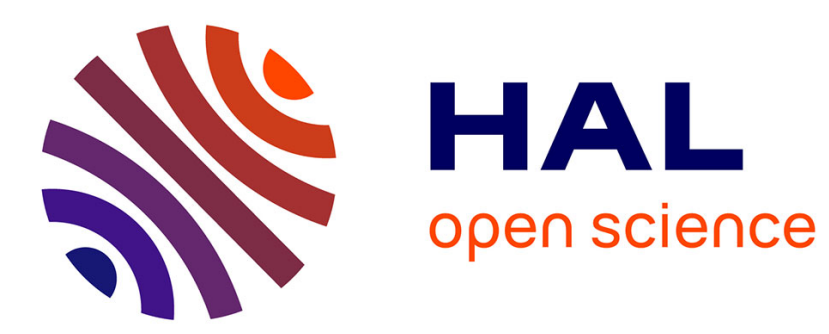

\title{
Experimental Evidence for a High-Pressure Isostructural Phase Transition in Osmium
}

Florent Occelli, Daniel L Farber, James M Badro, Chantel M Aracne, David M Teter, Michael M Hanfland, Bernard M Canny, Bernard M Couzinet

\section{To cite this version:}

Florent Occelli, Daniel L Farber, James M Badro, Chantel M Aracne, David M Teter, et al.. Experimental Evidence for a High-Pressure Isostructural Phase Transition in Osmium. Physical Review Letters, 2004, 93, pp.095502. 10.1103/PhysRevLett.93.095502 . insu-01387809

\section{HAL Id: insu-01387809 https://hal-insu.archives-ouvertes.fr/insu-01387809}

Submitted on 26 Oct 2016

HAL is a multi-disciplinary open access archive for the deposit and dissemination of scientific research documents, whether they are published or not. The documents may come from teaching and research institutions in France or abroad, or from public or private research centers.
L'archive ouverte pluridisciplinaire HAL, est destinée au dépôt et à la diffusion de documents scientifiques de niveau recherche, publiés ou non, émanant des établissements d'enseignement et de recherche français ou étrangers, des laboratoires publics ou privés. 


\title{
Experimental Evidence for a High-Pressure Isostructural Phase Transition in Osmium
}

\author{
Florent Occelli, ${ }^{1}$ Daniel L. Farber, ${ }^{1}$ James Badro, ${ }^{1,2}$ Chantel M. Aracne, ${ }^{1}$ David M. Teter,${ }^{3}$ Michael Hanfland, ${ }^{4}$ \\ Bernard Canny, ${ }^{5}$ and Bernard Couzinet ${ }^{5}$ \\ ${ }^{1}$ Earth Science Division and IGPP, Lawrence Livermore National Laboratory, USA \\ ${ }^{2}$ Laboratoire de Minéralogie Cristallographie, Institut de Physique du Globe de Paris, France \\ ${ }^{3}$ Organization 9753, Sandia National Laboratory, USA \\ ${ }^{4}$ European Synchrotron Radiation Facility, France \\ ${ }^{5}$ Laboratoire de Physique des Milieux Condensés, Université Paris VI, France \\ (Received 4 December 2003; revised manuscript received 25 February 2004; published 24 August 2004; \\ corrected 26 August 2004)
}

\begin{abstract}
We have measured the isothermal equation of state (EOS) of osmium to $75 \mathrm{GPa}$ under hydrostatic conditions at room temperature using angle-dispersive $\mathrm{x}$-ray diffraction. A least-squares fit of this data using a third-order Birch-Murnaghan EOS yields an isothermal bulk modulus $K_{0}=411 \pm 6 \mathrm{GPa}$, showing osmium is more compressible than diamond. Most importantly, we have documented an anomaly in the compressibility around $25 \mathrm{GPa}$ associated with a discontinuity in the first pressure derivative of the $c / a$ ratio. This discontinuity plausibly arises from the collapse of the small holeellipsoid in the Fermi surface near the $L$ point.
\end{abstract}

DOI: $10.1103 /$ PhysRevLett.93.095502

Recently, there has been much interest the highpressure properties of zinc and most specifically the nature of a possible Lifshitz [1] or electronic topological transition (ETT) near $10 \mathrm{GPa}$. This type of phase transition arises when distortion of the electronic band structure results in a topological singularity of the Fermi surface. Interestingly, while the experimental data remain somewhat ambiguous [2-5], simulations suggest an ETT indeed exists in this pressure range [6-8]. Recently, Steinle-Neumann et al. [8] have suggested that the transition arises from changes in the band structure near the high-symmetry point $K$, where three bands cross the Fermi surface upon compression. This suggests one might expect other hcp metals to exhibit similar phenomena. The platinoid $5 d$ transition elements (e.g., Re, Os, and Ir) are known to be the densest and stiffest metals $[9,10]$, suggesting they might in fact be poor candidates in which to look for such effects. However, experimental and theoretical results for Os $[11,12]$ have shown the existence of small local maxima in the band structure just above the Fermi energy near the $L$ point on the zone boundary [11]. Thus, the potential exists that these structures might upon compression fall below the Fermi energy and give rise to an ETT. Furthermore, recent equation of state (EOS) measurements by Cynn et al. [13] have suggested that Os has a bulk modulus $\left(K_{0}\right)$ and first derivative of the bulk modulus $\left(K_{0}^{\prime}\right)$ equal to $462 \mathrm{GPa}$ and 2.4 , respectively, and is less compressible than diamond $\left(K_{0}=446 \mathrm{GPa}\right.$ and $K_{0}^{\prime}=3$ ) [14]. This conclusion has strong implications for the nature of the metallic bond in Os and paradoxically implies that the latter, where bonding electrons are delocalized, is less compressible than the covalent bond, where bonding electrons are localized. The difficulty in supporting such a claim, however, arises due to the fact that in EOS studies and especially those of low-
PACS numbers: 61.66.Bi, 07.35.+k, 62.20.Dc, 61.10.Nz

compressibility materials, where the maximum experimental pressures are only a small fraction of the value of $K_{0}$, there exists a strong inverse correlation between the values of $K_{0}^{\prime}$ and those of $K_{0}$ in the numerical inversion of the volume as a function of pressure data. In order to accurately investigate the magnitude of $K_{0}$ and $K_{0}^{\prime}$ simultaneously in materials such as Os, one needs to undertake a high-resolution study in the low-pressure region $\left(K_{0}\right)$ and also obtain high-pressure data to constrain $K_{0}^{\prime}[15]$.

In order to clarify these points, we have undertaken an experimental study of the EOS of osmium using angledispersive $\mathrm{x}$-ray diffraction with ultrahigh accuracy. Great care was taken to compress the samples hydrostatically to obtain the most accurate pressure measurements and to minimize both the stress distribution on the sample and the error in the bulk modulus. Pressure on the sample was measured by ruby fluorescence before and after the $\mathrm{x}$ ray diffraction data were collected to ensure there was no pressure drift during the measurement. Of great help in that endeavor was the extremely short acquisition time ( $\sim 4 \mathrm{~s}$ of $\mathrm{x}$-ray exposure per diffraction pattern) available on third generation synchrotron sources. The samples (commercially available osmium with a 325 mesh) were loaded in three separate membrane actuated diamond anvils cells (DAC) and one symmetrical Mao-Bell-type DAC. One cell was set up for very high resolution in the low-pressure regime (up to $26 \mathrm{GPa} ; 0.5 \mathrm{GPa}$ per point) to better constrain $K_{0}$. In this cell, we used $350 \mu \mathrm{m}$ diamond culets, neon as a pressure transmitting medium, and a stainless-steel gasket [16]. The remaining cells were optimized for higher pressure experiments (up to $75 \mathrm{GPa}$ ) by using $150 \mu \mathrm{m} \times 350 \mu \mathrm{m}$ beveled diamond culets, helium as the pressure transmitting medium, and rhenium gaskets [16]. In our low-pressure run, pressure was measured using both the $\mathrm{NaCl}$ EOS by placing a small amount of 
$\mathrm{NaCl}$ in the sample chamber as an internal pressure marker and by ruby fluorescence [17]. In the highpressure runs, pressure was measured using only ruby fluorescence. Above the B1-B2 phase transition at $29 \mathrm{GPa}$ [18], $\mathrm{NaCl}$ can no longer be used reliably as an internal pressure standard.

Angle-dispersive $\mathrm{x}$-ray diffraction measurements were recorded at ID09A of the European Synchrotron Radiation Facility, Grenoble, France [19]. A total of 33 diffraction patterns were recorded between 0 and $20 \mathrm{GPa}$, and 10 more points between 20 and $75 \mathrm{GPa}$. Figure 1 shows typical diffraction patterns from our experiment; the narrow diffraction lines even at the highest pressure indicate the near absence of nonhydrostatic stresses and pressure drift during the measurement. No less than 12 reflections from osmium are resolved on each pattern, enabling us to minimize the errors on the lattice parameters using a crystal structure refinement procedure. The Rietveld structural refinements were performed using GSAS (General Structural Analysis System) [20]. Over this pressure range, our data confirm that no first order structural phase transition occurs. The errors on the volumes derived from our data are at most $5 \times 10^{-3} \mathrm{~A}^{3}$.

Our new EOS is shown in Fig. 2 using the third-order Birch-Murnaghan [21] equation; $P=3 / 2 K_{0}\left[\left(V / V_{0}\right)^{-7 / 3}-\right.$ $\left.\left(V / V_{0}\right)^{-5 / 3}\right]\left\{1+3 / 4\left(K_{0}^{\prime}-4\right)\left[\left(V / V_{0}\right)^{-2 / 3}-1\right]\right\}$, where $V$ is the volume, $V_{0}$ is the zero pressure volume, $K_{0}$ is the isothermal bulk modulus, and $K_{0}^{\prime}$ is the first pressure derivative of the isothermal bulk modulus. The leastsquares fit to our data yields $K_{0}=411 \pm 6 \mathrm{GPa}, K_{0}^{\prime}=$ $4.0 \pm 0.2$, and $V_{0}=27.941 \pm 0.008 \mathrm{~A}^{3}$. Using a stochastic minimization algorithm on the third-order BirchMurnaghan EOS, which gives a more robust estimate of the parameters as well as the correlation matrix, we find $K_{0}=410.9 \pm 1.2 \mathrm{GPa}, \quad K_{0}^{\prime}=3.97 \pm 0.04, \quad$ and $V_{0}=$ $27.949 \pm 0.002 \mathrm{~A}^{3}$. Further, the refined values of $V_{0}$ in osmium obtained in this study are equal to the reference

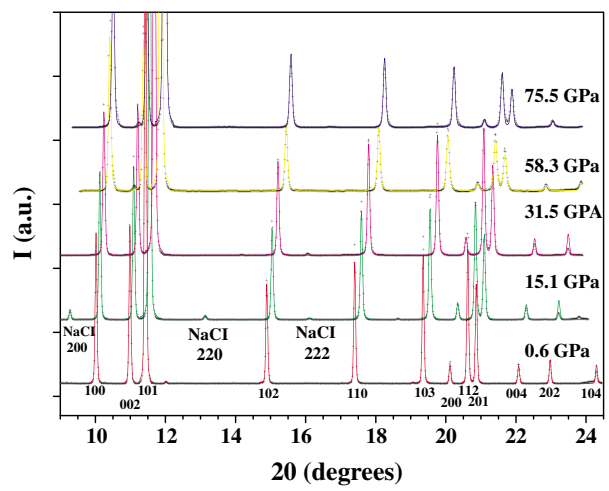

FIG. 1 (color online). Integrated angle-dispersive diffraction patterns recorded at various pressures. The experimental data are represented by a + symbol, and the curves are obtained from a Rietveld structural refinement of the data. The osmium reflections are labeled on the lowermost spectrum. $\mathrm{NaCl}$ reflections are labeled on the second spectrum. value given by ICSD (Inorganic Crystal Structure Database) $\left(V_{0}=27.958 \mathrm{~A}^{3}\right)$ within $2 \sigma$, and our new results show that contrary to the conclusions of Cynn et al. [13], the bulk modulus of osmium is actually $8 \%$ smaller than that of diamond, with $K_{0}^{\prime}$ close to 4 as is expected for a well-behaved incompressible material. Indeed, the most surprising result of the previous study of Cynn et al. [13] was the extremely low value of $K_{0}^{\prime}$ in Os $\left(K_{0}^{\prime}=2.4\right)$ obtained using a third-order BirchMurnaghan EOS. Indeed, theory suggests that this value should be close to 4 in stiff materials [21]. Furthermore, the Birch-Murnaghan formulation itself is valid only where this value is sufficiently close to 4 . To date then, diamond remains the most incompressible material known.

As a further check on our experimental results, we have performed first-principles pseudopotential planewave total-energy calculations within the local density (LDA) and generalized gradient approximations (GGAPW91) to electronic exchange and correlation on hcposmium and diamond-carbon crystal structures [2326]. Table I shows our new calculations, which agree with the theoretical results presented by Cynn et al. [13]. Interestingly, while our conclusions are at odds with those published by Cynn et al. [13], they are in qualitative agreement with the theoretical calculations presented in that same study. In the latter, the theoretical bulk modulus of osmium was determined to be lower than the theoretical bulk modulus of diamond (cf. Table I), and $K_{0}^{\prime}$ is in good agreement with our new experimental and theoretical results. The explanation of the differences in the conclusions of the present study with those of Cynn et al. [13] stems from the fundamentally different experimental approaches taken in the two studies. In their work on the EOS of osmium, Cynn et al. [13] used energy dispersive $\mathrm{x}$-ray diffraction. This technique relies on the

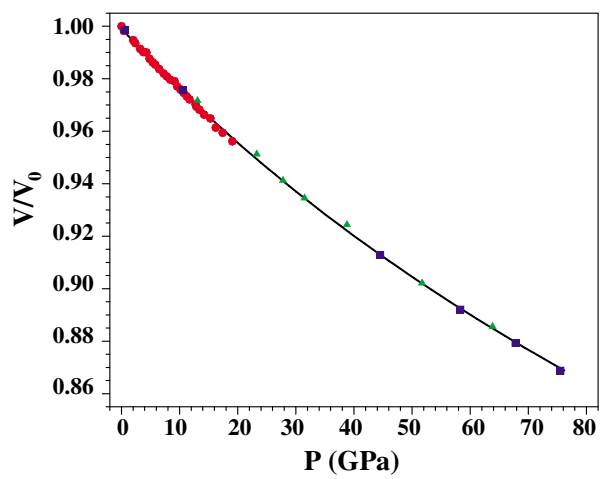

FIG. 2 (color online). The isothermal equation of state of Osmium at $300 \mathrm{~K}$. The different symbols correspond to the three independent loadings (the size of the symbol is greater than the experimental errors both on pressure and volume). The solid curve is a third-order Birch-Murnaghan fit to the data yielding $K_{0}=411 \pm 6 \mathrm{GPa}, \quad K_{0}^{\prime}=4.0 \pm 0.2, \quad$ and $\quad V_{0}=$ $27.941 \pm 0.008 \mathrm{~A}^{3}$. 
TABLE I. Third-order Birch-Murnaghan isothermal EOS parameters for osmium and diamond from various studies.

\begin{tabular}{llcccc}
\hline \hline & & Osmium & & Diamond & \\
& & $K_{0}(\mathrm{GPa})$ & $K_{0}^{\prime}$ & $K_{0}(\mathrm{GPa})$ & $K_{0}^{\prime}$ \\
\hline \multirow{4}{*}{ This study } & exp & 411 & 4.0 & & \\
& LDA & 437.3 & 4.46 & 464.0 & 3.65 \\
& GGA & 382.3 & 4.60 & 431.2 & 3.68 \\
Cynn et al. & exp & 462 & 2.4 & & \\
Occelli et al. & LDA & 444.8 & 4.4 & 467.1 & 3.6 \\
\hline \hline
\end{tabular}

use of an intense white beam, and while it was necessary for high-pressure diamond anvil experiments on second generation synchrotron sources, it results in low-accuracy volume refinements which are the critical parameter in EOS studies and even more crucial in the study of lowcompressibility materials. Poor resolution on the volume refinements is further exacerbated by the use of stiff pressure media (such as argon above $30 \mathrm{GPa}$ ) that produce large pressure gradients and thus increase the error bounds. The quantitative discrepancy between these two studies, albeit small, does have drastic consequences on the physical interpretation of the nature of the chemical bond in osmium.

Aside from the straightforward determination of the EOS of osmium, our new data present a most surprising and novel result; although a simple analysis of the EOS indicates that there are no first order structural transitions, a more detailed analysis of the $c / a$ ratio as a function of pressure [Fig. 3(a)] shows an interesting fea- ture around $25 \mathrm{GPa}$. Two distinct compression regimes can be identified, below and above $\sim 25 \mathrm{GPa}$. Attempts to fit $c / a$ versus pressure with either a single linear, quadratic, or cubic function all yielded highly correlated errors. Only a two-piece linear fit provides a statistically valid description of the data. Importantly, these two regimes are not resolvable as a function of volume [Fig. 3(b)] but only as a function of pressure [Fig. 3(a)]. When plotted as a function of volume, the $c / a$ ratio exhibits a monotonically increasing behavior [Fig. 3(b)] and can be approximated by a unique linear function. This means that the modulus, namely $\delta P / \delta V$, must also exhibit the same discontinuity as seen in the function $c / a(P)$. This further implies that $V(P)$ should also display this anomaly, and, in that respect, two independent BirchMurnaghan EOS were fitted to the data below and above $25 \mathrm{GPa}$, as reported in Fig. 2. In that case, the reduced $\chi^{2}$ drops by $60 \%$, and using an $F$-test comparison the twophase model is statistically significant at the $96 \%$ confidence level, strengthening our assumption (for a phase transition) from a statistical standpoint. It is noteworthy that in the latter case, both bulk moduli $(392 \pm 2 \mathrm{GPa}$ and $400 \pm 5.5 \mathrm{GPa}$ for low-pressure and high-pressure data, respectively) are still lower than that of diamond, and actually lower (but within error bars) than the one obtained with one EOS fit of the entire data set.

These structural changes likely arise from changes in the electronic structure on compression and could be the signature of a Lifshitz transition, where the first derivative of the compressibility presents a discontinuity at the transition pressure and the second derivative is not defined. Such transitions arise by passage of the Fermi surface through a topologic singularity [1]. In the most
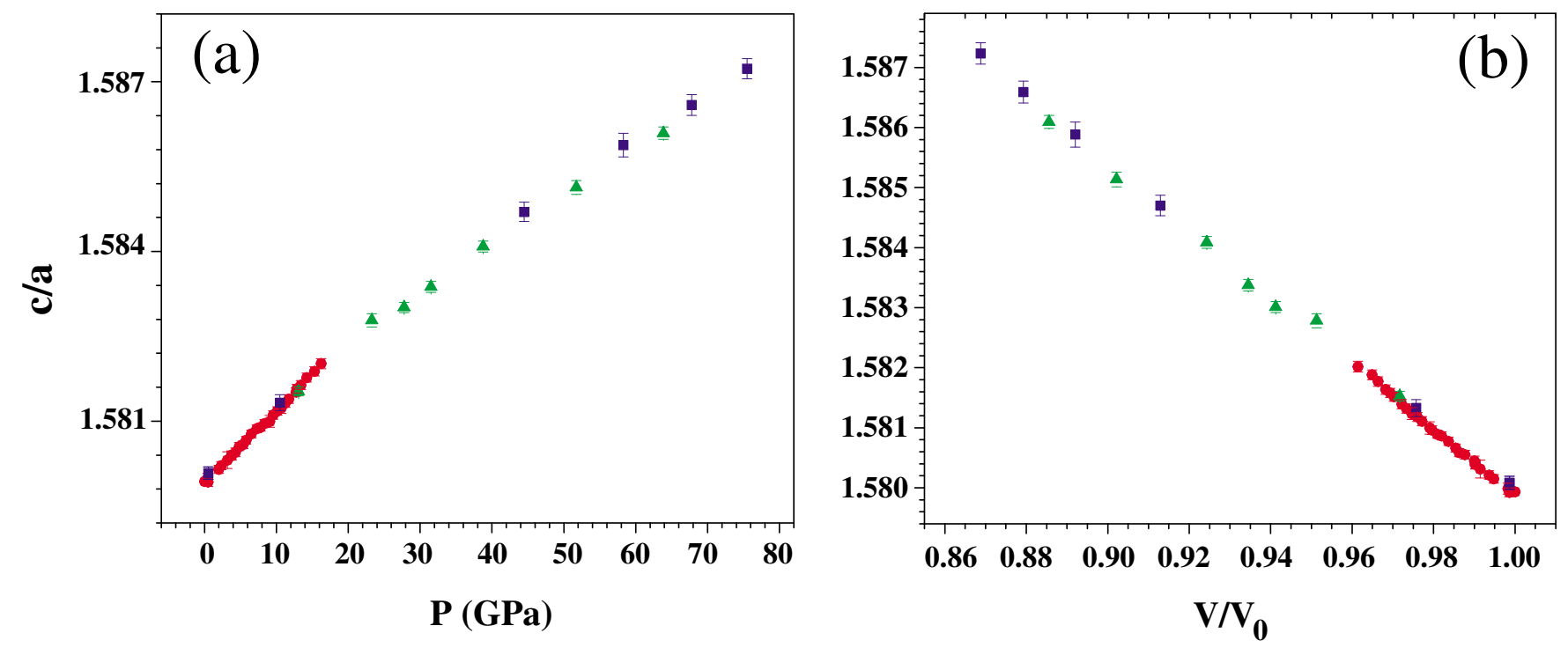

FIG. 3 (color online). The $c / a$ ratio of osmium versus pressure (a) and volume (b). The symbols are the same as in Fig. 2 . The linear fits to the $c / a$ versus pressure data yield $c / a=1.57993+1.25049 \times 10^{-4} P+O\left(P^{2}\right)$ over the pressure range 0 to $25 \mathrm{GPa}$ and $c / a=1.58071+8.65348 \times 10^{-5} P+O\left(P^{2}\right)$ from $25 \mathrm{GPa}$ to the highest pressure achieved in the study. The linear fit to the $c / a$ versus reduced volume data yields $c / a=1.63504-0.0552\left(V / V_{0}\right)+O\left(V^{2}\right)$. 
studied example of zinc [8], recent calculations show that the transition arises from changes in the band structure near the high-symmetry point $K$ where three bands, which lie above the Fermi level at zero pressure, cross the Fermi level upon compression. In the case of osmium, we suggest that the anomaly likely arises from the passage of the maxima in band $h_{7}$ below the Fermi energy with compression resulting in the collapse of the small hole ellipsoid. While nontrivial, this hypothesis can now be experimentally verified either by single-crystal highpressure inelastic $\mathrm{x}$-ray scattering measurements or de Haas-van Alphen measurements with recently developed diamond anvil techniques [27,28]. Finally, our findings prove the importance of ultrahigh accuracy measurements in the lattice constants in conjunction with close to ideal pressure conditions and shows how these types of studies can help pinpoint, isolate, and even unveil peculiar behaviors in materials that could otherwise not be observed.

We thank Jagan Akella, Michael Krisch, Kevin Moore, and Quentin Williams for helpful reviews. We acknowledge the European Synchrotron Radiation Facility for provision of synchrotron radiation facilities. Work performed under the auspices of the U.S. Department of Energy by the Lawrence Livermore National Laboratory under Contract No. W-7405-ENG-48.

[1] I. M. Lifshitz, Zh. Eksp. Teor. Fiz. 38, 1569 [Sov. Phys. JETP 11, 1130 (1960)].

[2] K. Takemura, Phys. Rev. B 56, 5170 (1997).

[3] K. Takemura, Phys. Rev. B 60, 6171 (1999).

[4] S. Klotz, M. Braden, and J. M. Besson, Phys. Rev. Lett. 81, 1239 (1998).

[5] A. B. Garg, V. Vijayakumar, P. Modak, D. M. Gaitonde, R. S. Rao, B. K. Godwal, and S. K. Sikka, J. Phys. Condens. Matter 14, 8795 (2002).

[6] D. L. Novikov， M. I. Katsnelson，A.V. Trefilov，A. J. Freeman, N.E. Chirstensen, A. Svane, and C.O. Rodriguez, Phys. Rev. B 59, 4557 (1999).

[7] L. Fast, R. Ahuja, L. Nordström, J. M. Wills, B. Johansson, and O. Eriksson, Phys. Rev. Lett. 79, 2301 (1997).

[8] G. Steinle-Neumann, L. Stixrude, and R. Cohen, Phys. Rev. B 63, 054103 (2001).

[9] J. Friedel, in The Physics of Metals. I. Electrons, edited by J.M. Ziman (Cambridge University Press, Cambridge, 1969), p. 340.

[10] M. E. Fine, L. D. Brown, and H. L. Marcus, Scr. Metall. 18, 951 (1984).

[11] G. N. Kamm and J. R. Anderson, Phys. Rev. B 2, 2944 (1970).

[12] V. I. Smelyansky, A. Perlov, and V. N. Antonov, J. Phys. Condens. Matter 2, 9373 (1990).

[13] H. Cynn, J. E. Klepeis, C.-S. Yoo, and D. A. Young, Phys. Rev. Lett. 88, 135701 (2002).
[14] F. Occelli, P. Loubeyre, and R. Le Toullec, Nature Materials 2, 151 (2003).

[15] W.-B. Holzapfel, Z. Kristallogr. 216, 473 (2001).

[16] Note the freezing point of neon at room temperature is at 4.7 GPa. [L.W. Finger, R. M. Hazen, G. Zou, H. K. Mao, and P. M. Bell, Appl. Phys. Lett. 39, 892 (1981)]; The freezing point of helium at room temperature is $11.5 \mathrm{GPa}$. [J. Besson and P. Pinceaux, Science 206, 1073 (1979)].

[17] H. K. Mao, J. Xu, and P. M. Bell, J. Geophys. Res. B 91, 4673 (1986); C. S. Smith and L. S. Cain, J. Phys. Chem. Solids 36, 205 (1975).

[18] W. A. Bassett, T. Takahashi, H.-K. Mao, and J. S. Weaver, J. Appl. Phys. 39, 319 (1968).

[19] The X-ray beam was focused vertically with a Pt-covered Si mirror and horizontally with an asymmetrically cut bent $\mathrm{Si}(111)$ Laue monochromator to a spot size of $15 \times$ $15 \mu \mathrm{m}$ FWHM. The energy was fixed at $30.006 \mathrm{keV}$, and the sample-to-detector distance was $362.6184 \mathrm{~mm}$. The images were recorded in $4 \mathrm{~s}$ using a two-dimensional MAR345 detector and integrated using the FIT2D software package. (J. Hammersley, ESRF Report No. ESRF98HAO1T, 1996.)

[20] A. C. Larson and R. B. Von Dreele, Los Alamos National Laboratory Report No. LAUR 86, 2000.

[21] For example, J. P. Poirier, Introduction to the Physics of the Earth's Interior (Cambridge University Press, Cambridge, 1991). In order to test the robustness or our inversion for the bulk modulus and its first pressure derivative, we have also fit the data with the formalism of Vinet et al. [22]. This fit yields $K_{0}=410 \pm 6 \mathrm{GPa}$, $K_{0}^{\prime}=4.2 \pm 0.2$, and $V_{0}=27.942 \pm 0.009 \mathrm{~A}^{3}$, in excellent agreement with those derived using the third-order Birch-Murnaghan equation.

[22] P. Vinet, J. R. Smith, J. Ferrante, and J. H. Rose, Phys. Rev. B 35, 1945 (1987).

[23] Calculations were performed using the Vienna ab initio simulation program (VASP) developed at the Technische Universitat Wien.

[24] G. Kresse and J. Hafner, Phys. Rev. B 47, 558 (1993); 49, 14251 (1994); G. Kresse and J. Furthmuller, Comput. Mater. Sci. 6, 15 (1996); Phys. Rev. B 54, 11169 (1996).

[25] J. P. Perdew et al., Phys. Rev. B 46, 6671 (1992).

[26] Vanderbilt ultrasoft pseudopotentials were generated for osmium and carbon. The electronic degrees of freedom were minimized using a preconditioned conjugategradient method and cell parameters for the hcp-osmium were optimized at all volumes. A plane-wave energy cutoff of $400 \mathrm{eV}$ was found to sufficient converge the energies of both the osmium and carbon structures. A Monkhorst-Pack grid of $[16,16,10]$ was used for hcposmium and $[4,4,4]$ was used for diamond-carbon. Bulk moduli were thus calculated using a third-order BirchMurnaghan isothermal equation of state.

[27] D. Jackson, C. Aracne-Ruddle, V Malba, S. Weir, S. Catledge, and Y. Vohra, Rev. Sci. Instrum. 74, 2467 (2003).

[28] S Gilder, M. LeGoff, J. Peyronneau, and J. Chervin, Geophys. Res. Lett. 29, 1392 (2002). 